\title{
Utility-based Recommender Systems Using Implicit Utility and Genetic Algorithm
}

\author{
Deng Feng \\ College of Economics and Management \\ Beijing University of Technology \\ Beijing, China \\ Dfx7799@sina.com
}

\begin{abstract}
Recommender systems are used to recommend items for user in e-commerce with information overload. Utility-based recommender systems build multi-attribute utility function of user and recommend the highest utility item for user. Some utility-based recommender systems use rating for items to extract utility function, which produce significant burden for user. The paper proposes a utility-based recommender technique which can predict attribute value utility and implicit holistic utility rate of items by user browsing behavior and genetic algorithm, and elicit the attribute weight by genetic algorithm, and building a multi-attribute utility function. The experimental results on clothing recommendation show that the proposed method is superior to current utility-based methods on accuracy, satisfaction, usefulness and time expense.
\end{abstract}

Keywords-recommended system; multi-attribute utility; implicit utility; genetic algorithm; browsing behavior

\section{I . INTRODUCTION}

Recommender systems can help customers search for the products which they like in e-commerce context. Utility-based recommender systems can extract the decision maker's utility function, and calculate the utility of each item, and recommend high-utility items to users $[1,2]$. Utility-based recommender systems can improve rating sparsity, new items, new users problems that collaborative filtering recommender technique encounters [3], and it outperforms the traditional content-based recommender systems [1].

The central technique of utility-based recommender systems is constructing utility function. Much utility function is based on multi-attribute utility theory (MAUT). A customer makes buying decision based on multiple decision attribute of products. MAUT uses multi-attribute utility function to model customer preference, and maximize utility function to look for the best decision on the basis of multi-criteria. Some utility function extraction techniques are able to come to high decision accuracy, such as simple multi-attribute rating technique exploiting ranks (SMARTER), radial basis function network (RBFN) [4], regression analysis [5,6], and neural network.
However, these methods require user rating and increase user effort.

This paper designs a utility function extraction technique which is based on MAUT and use implicit utility from user browsing behaviors data [7] and genetic algorithm (GA) [8,9], and which is called IU-GA. A utility-based recommender system is proposed based on IU-GA which can classify item data based on central item attributes, and calculate utility of items with user preferring attributes and recommend top-N utility items to user.

\section{II . LITERATURE REVIEW}

This section briefly introduces utility-based recommender systems, MAUT, and implicit utility elicitation methods.

\section{A. Utility-based recommender systems}

Utility-based recommender systems make recommendations based on the calculation of the utility of each item for the user. Utility-based recommender techniques elicit multi-attribute utility function based on item rates that user offer to describe user preferences, and apply the utility function to calculate item utility for user [3]. Utility-based recommendations have not some problems of collaborative filtering recommender technique, such as cold-start, sparsity, and high dimensions.

However, utility-based recommender systems face eliciting utility function for each user, which require remarkable burden of interaction. Several utility-based recommender systems have been developed based on user rating for items [4-6,10,11]. They are based on MAUT and needs significant user effort. So, modeling utility function with little user effort is a critical issue in designing utility-based recommender systems.

\section{B. Multi-attribute utility theory}

MAUT is a quantitative and systematical method to describe use making decision. MAUT can model user making-decision involving multiple interdependent objectives on the basis of uncertainty and preference analysis. A MAU function based on MAUT can be generally represented by: 


$$
\operatorname{MAU}\left(u_{1}, \Lambda, u_{j}, \Lambda, u_{m}\right)=\sum_{j=1}^{m} w_{j} u_{j}
$$

Where the number of attribute is $\mathrm{m}, \quad u_{j}$ is a single-attribute utility function about attribute $j, w_{j}$ is the weight for attribute $j$ and $\sum_{j=1}^{m} w_{j}=1\left(0 \leq w_{j} \leq 1\right)$.

\section{Implicit utility extraction}

Item rating that user has provided is explicit rating, which is used by much recommendation systems. However, explicit rating requires user effort and users are not willing to rate items. Therefore, implicit rating is proposed to substitute explicit rating. Implicit rating is elicited on the basis of user behavior, such as buying, collecting, browsing, clicking, and so on [12]. There is a positive correlation between browsing time and explicit rating $[7,13]$. So, implicit rating could be predicted by browsing time.

\section{PROPOSED RECOMMENDATION APPROACH}

In this section, firstly the system framework is presented. Then recommendation mechanism is described in detail step by step.

\section{A. Proposed system framework}

Firstly, implicit utility of each attribute value is elicited by its frequency in all items which user has browsed. Secondly, implicit holistic utility of each item which user has browsed is predicted by its browsing time. The weight of each attribute is calculated by genetic algorithm (GA). Based each attribute value utility and the weight of each attribute, MAU function is proposed and is used to calculate utility of all items including user preferring attribute value. So the proposed recommended technique is called IU-GA recommendation system which its system framework is showed by Fig .1.

\section{B. Multi-attribute utility model}

Assume that a item has $\mathrm{m}$ attributes and could be encoded into vector: $\quad I\left(a_{i 1}, a_{i 2}, \Lambda, a_{i j}, \Lambda, a_{i m}\right)$, where $I$ is a item vector and $a_{i j}$ is the value of attribute $j$ of item $I$. Assume that attribute $j$, where $1 \leq j \leq m$, has $l_{j}$ nominal values which are denoted by $V_{j}\left\{v_{j 1}, \Lambda, v_{j k}, \Lambda, v_{j l_{j}}\right\}$, where $V_{j}$ is a value collect of attribute $j$, and $v_{j k}$ is a attribute value. For a numerical attribute, its value is transformed to nominal value based on rank. For example, price is transformed to several ordinal scale and becomes nominal value. Assume clothing has four attributes: style, color, pattern, price. Table 1 shows the attribute values.

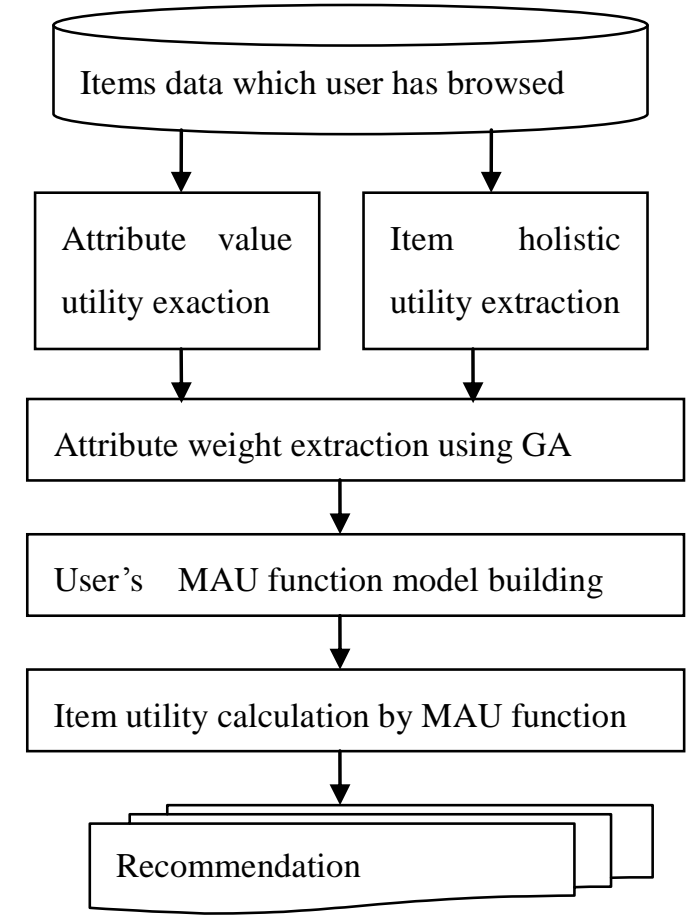

Figure 1. System framework

TABLE I. ATtRIBUtes AND VALUE RANGES OF Clothing

\begin{tabular}{|l|l|}
\hline Attribute & \multicolumn{1}{|c|}{ Value range } \\
\hline Style & Fresh, Sweet, Casual, ... \\
Color & White, Red, Green, ... \\
Pattern & Stripes, Polka dots, Square space, ... \\
Price & One(0-100), Two(100-200), Three(200-400), ... \\
\hline
\end{tabular}

Assume that attribute $\mathrm{j}$ of a item has only a value and the $u_{j}\left(v_{j k}\right)$ is the utility of attribute value $v_{j k}$. For a user, $u_{j}\left(v_{j k}\right)$ could be predicted by user behavior. Therefore, the utility of item $I$ for a user is gained based on MAUT. The MAU function is defined as follows:

$M A U_{i}=\sum_{j=1}^{m} w_{j} u_{j}\left(a_{i j}\right) \quad a_{i j} \in V_{j}\left\{v_{j 1}, \Lambda, v_{j l_{j}}\right\}$

Where $M A U_{i}$ is the holistic utility of item $I$, and $w_{j}$ is the weight for attribute $j$ for a user which shows the user preference of attribute $j$, and $\sum_{j=1}^{m} w_{j}=1\left(0 \leq w_{j} \leq 1\right)$.

\section{The implicit utility of attribute value}

The traditional recommender systems require users to rate for the utility $u_{j}\left(v_{j k}\right)$ of attribute value $v_{j k}$ which produces significant burden for users. Based on user 
browsing behavior, $u_{j}\left(v_{j k}\right)$ could be predicted. Assuming a user has browsed $\mathrm{n}$ items and $f_{j k}$ is the emerging frequency of value $v_{j k}$, which illustrates the number of items with attribute value $v_{j k}, u_{j}\left(v_{j k}\right)$ is predicted by:

$$
u_{j}\left(v_{j k}\right)=\frac{f_{j k}-\min _{1 \leq k \leq l_{j}}\left(f_{j k}\right)}{\max _{1 \leq k \leq l_{j}}\left(f_{j k}\right)-\min _{1 \leq k \leq l_{j}}\left(f_{j k}\right)}
$$

\section{The implicit holistic utility of item}

The implicit holistic utility of item is predicted by its user-browsing time. Assume $t_{i}$ is the time that user spends in browsing item $I$, and $u_{i}$ is the holistic utility of item $I$, which is predicted by:

$$
u_{i}=\frac{t_{i}-\min _{1 \leq p \leq n}\left(t_{i}\right)}{\max _{1 \leq i \leq n}\left(t_{i}\right)-\min _{1 \leq i \leq n}\left(t_{i}\right)}
$$

\section{E. The weight of attribute}

Based on the weight of every attribute, the utility of every attribute value, the holistic utility $M A U_{i}$ of item $I$ could be calculated on basis of MAU function. There should be a little difference between $M A U_{i}$ and $u_{i}$ predicted by browsing time. When there is least difference between $M A U_{i}$ and $u_{i}$ of all items which user has browsed, the weight of all attributes is fitted. Therefore, nonlinear programming problem is used to confirm the weight of all attributes. Assuming that $\min f\left(w_{1}, \Lambda, w_{m}\right) \quad$ is objective function, nonlinear programming model is defined as follows:

$$
\left\{\begin{aligned}
& \min f\left(w_{1}, \Lambda w_{m}\right)=\min \sum_{i=1}^{n}\left(u_{i}-M A U_{i}\right)^{2} \\
&=\min \sum_{i=1}^{n}\left(u_{i}-\sum_{j=1}^{m} w_{j} u\left(a_{i j}\right)\right)^{2} \\
& \sum_{j=1}^{m} w_{j}=1 \\
& 0 \leq w_{j} \leq 1, \quad j=0, \Lambda, m
\end{aligned}\right.
$$

The genetic algorithm (GA) is used for optimization of objective function. The value of each weight is continuous and also between 0 and 1, 1/100th precision is made for each attribute weight by 7 bit. Fitness function is $f\left(w_{1}, \Lambda, w_{m}\right)$, and stochastic tournament method is used for selection operation, and two-point crossover operator is used for crossover operation to produce offspring, and selecting some elements of individual and changing its value are for mutation operation, and the number of iteration is 100 .

\section{F. Final recommendation}

When the weight of each attribute and the utility of each attribute value have been predicted, the holistic utility of each item is calculated by MAU function. If the number of items in the database is very large, the time expense is very remarkable. So, attribute values of items that user has browsed compose user-preferring attribute-value collect (PAVC), and items which attribute value belongs to PAVC could been recommended. For example, style attribute of clothing that user has browsed have two values: fresh and sweet, and the PAVC of style attribute is $\{$ Fresh, Sweet $\}$. Each attribute has PAVC that user prefers. Assume that PAVC of attribute $j$ is $P A V C_{j}$, and the items collect according to user preference is $I C$. $I C$ is denoted by:

$$
I C=\left\{I \mid a_{i j} \in P A V C_{j}, j=1, \Lambda, m\right\}
$$

The utility of items of $I C$ is calculated by MAC function, and items are ranked by their utility. In the end, the top 20-ranked items are recommended for user.

\section{EXPERIMENTS}

Huang (2011) investigated the performance of two utility-based recommendation techniques: SMARTER and RBFN. The experiments result testified that SMARTER is superior to RBFN on accuracy, satisfaction and usefulness for movie recommendation. If the performance of IU-GA is superior to SMARTER, IU-GA is considerable.

The dataset is composed of 1000 skirt data from China Taobao. Clothing has ten attributes: style (9 values), color (15 values), pattern (15 values), collar (6values), sleeve (10 values), waist (5 values), fabric (7 values), skirt shape (6 values), skirt length (4 values), fitting (3 value). 20 students of a university have taken part in the experiment. SMARTER recommended 20 items that are Group 1. IU-GA recommended 20 items that are Group 2. Every user rate for satisfaction and usefulness of two group items, which rating range is from 0 to 5 , and 5 is the most satisfying and useful. Every user selects items which could be bought and put them into the shopping car.

\section{A. Accuracy evaluation}

The precision and recall are most popular metrics that evaluate recommendation accuracy (Herlocker, et al., 2004; Salehi, et al., 2013)[9,14]. The precision is a measure of exactness and the recall is a measure of completeness. In this paper, the precision is calculated by:

$$
P=\frac{|P R E \cap R E|}{|R E|}
$$

Where $P$ is the precision, and $P R E$ is the collect of items which user prefers and are put into the shopping cart, and $R E$ is the collect of items which are recommended for user by recommended system. The recall $R$ is calculated by:

$$
R=\frac{|P R E \cap R E|}{|I C|}
$$

When the size of the recommendation set increases, 
the precision would decrease and the recall would increase. So, Fl is a combination metric which is denoted as follows:

$$
F 1=\frac{2 \times P \times R}{P+R}
$$

The result of recommendation accuracy for 20 students is shown in Fig .2, Fig .3 and Fig .4. Precision, recall and F1 of IU-GA are higher than SMARTER. That reveals accuracy of IU-GA is higher than SMARTER.

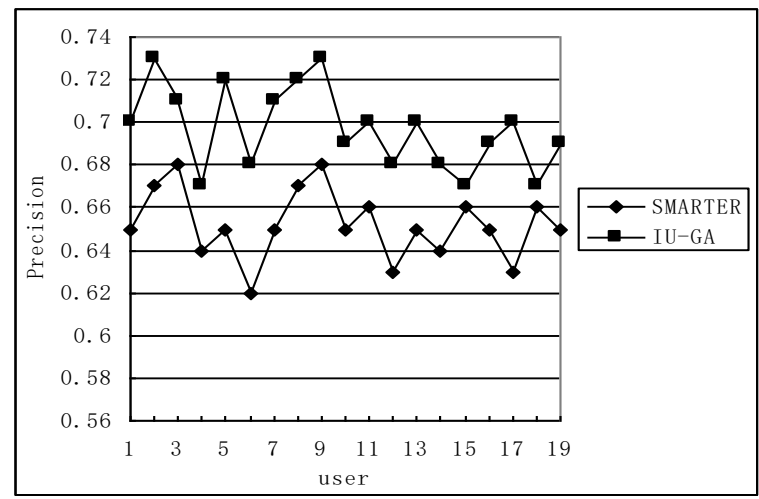

Figure 2. Precision of SMATER and IU-GA

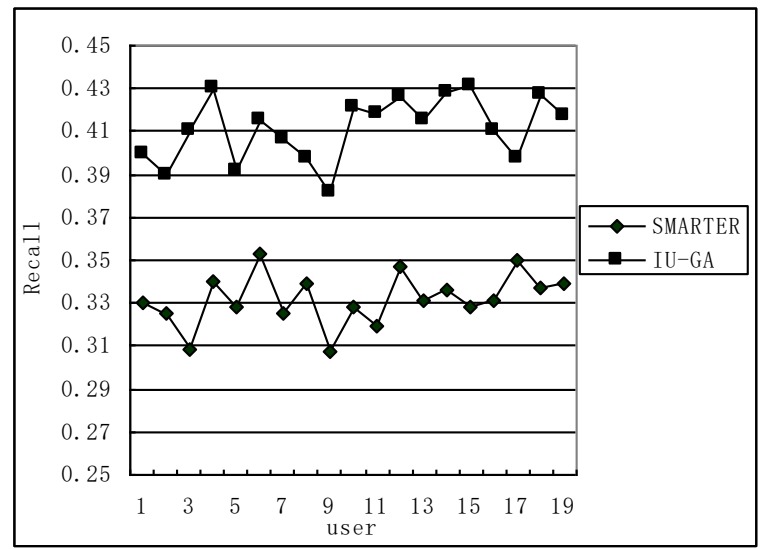

Figure 3. Recall of SMATER and IU-GA

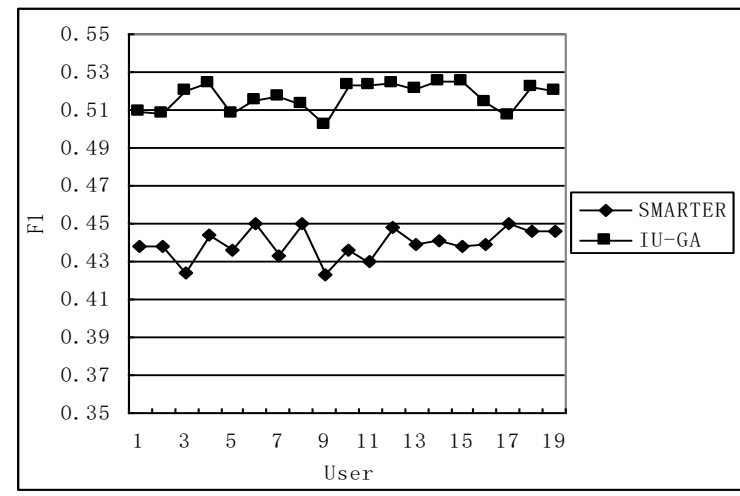

Figure 4. F1 of SMATER and IU-GA

\section{B. Performance evaluation}

Satisfaction, usefulness and time expense can be used to measure the performance of recommendation of SMARTER and IU-GA. The ANOVA results, shown in Table 2, reveal that there is significant difference on satisfaction, usefulness and time expense. Satisfaction and usefulness of items recommended by IU-GA is higher than that by SMARTER, time expense of IU-GA is less than time expense of SMARTER.

TABLE II. EFFECTS OF SMARTER AND IU-GA

\begin{tabular}{ccccc}
\hline Variable & $\begin{array}{c}\text { SMARTER } \\
\text { (Means) }\end{array}$ & $\begin{array}{c}\text { IU-GA } \\
\text { (Means) }\end{array}$ & F & Sig \\
\hline Satisfaction & 4.368 & 4.675 & 1.07 & .109 \\
Usefulness & 4.017 & 4.394 & 0.76 & .127 \\
$\begin{array}{c}\text { Time } \\
\text { expense }\end{array}$ & 320.9 & 164.4 & 2.49 & .118 \\
\hline
\end{tabular}

\section{CONCLUSIONS}

Utility-based recommended system attempts to model a user's multi-attribute utility function and recommend items with the highest utilities. The traditional utility--based recommended system require users to rate items to extraction utility function, which made more user burden and lower satisfaction. In the paper, the proposed recommended system (IU-GA) extracts multi-attribute utility function based on user browsing behavior and genetic algorithm, which requires less user effort. A laboratory experiment was conducted. Experiment result shows the performance of IU-GA is superior.

Because the proposed recommended system is only tested on clothing recommendation. This recommended system is limited to be used in online context and for user short-term interest. User long-term preference should be investigated by other utility--based recommended system based on user rating. Therefore, future studies can test the IU-GA performance on other items and focus in building multi-attribute utility function on user long-term preference.

\section{REFERENCES}

[1] S. 1. Huang, "Designing Utility-based Recommender Systems for E-commerce: Evaluation of Preference-elicitation Methods," Electronic Commerce Research and Applications, vol.10, 2011, pp.398-407.

[2] N. Manouselis and C. Costopoulou, "Analysis and Classification of Multi-criteria Recommender Systems," World Wide Web: Internet and Web Information Systems, vol 10, Apr. 2007, pp.415-441.

[3] R. Burke, "Hybrid Recommender System: Survey and Experiments," User Modeling and User-Adapted Interaction, vol. 12, 4, 2002, pp.331-370.

[4] F. R. Lin, S. L. Huang, Y. C. Yang, "Using Radial Basis Function Networks to Model Multi-attribute Utility Functions," In Proceedings of the 4th Workshop on e-Business, Las Vegas, USA, 2005.

[5] D. Jannach, Z. Karakaya, F. Gedikli, “Accuracy Improvements for Multi-criteria Recommender Systems," in: Proceedings of the 
13th ACM Conference on Electronic Commerce (EC 2012), 2012 pp.674-689.

[6] M.Nilashi, D. Jannach , O.Ibrahim, et al, "Clustering- and regression-based multi-criteria collaborative filtering with incremental updates," Information Sciences, vol. 293, 2015, pp.235-250.

[7] E. R.Núñez-Valdéz, J. M. Cueva-Lovelle, O. Sanjuán-Martı'nez, et al, "Implicit Feedback Techniques on Recommender Systems Applied to Electronic Books," Computers in Human Behavior vol. 28, Apr. 2012, pp.1186-1193.

[8] T. H.Dao, S. R. Jeong, H. A. Ahn, "Novel Recommendation Model of Location-based Advertising: Context-Aware Collaborative Filtering Using GA approach," Expert Systems with Applications, vol. 39, 2012, pp.3731-3739.

[9] M. Salehi, P. Mohammad, S. A. Razavi, "Hybrid Attribute-based Recommender System for Learning Material Using Genetic Algorithm and A Multidimensional Information Model,' Egyptian Informatics Journal ,vol.14, 2013, pp.67-78
[10] G. Adomavicius, Y. Kwon, "New Recommendation Techniques for Multi-Criteria Rating. Systems," IEEE Intelligent Systems, vol.22, Mar. 2007, pp.48-55.

[11] F. Hdioud, B. Frikh, B. Ouhbi, "Multi-Criteria Recommender Systems based on Multi-Attribute Decision Making," iiWAS2013, 2-4 December, 2013, Vienna, Austria, Copyright 2013 ACM.

[12] Nichols," Implicit Rating and Filtering," Proceedings of 5th DELOS Workshop on Filtering and Collaborative Filtering(Budapest), ERCIM, 1998, pp.31-36.

[13] Y. D. Choik, G. Kim, et al. "A Hybrid Online-product Recommendation System: Combining Implicit Rating-based Collaborative Filtering and Sequential Pattern Analysis," Electronic Commerce Research and Applications, vol.11, 2012, pp.309-317.

[14] J. L. Herlocker, J. A. Konstan, L. G. Terveen, et al, "Evaluating Collaborative Filtering Recommender Systems," ACM TRAns Inform Syst(TOIS), vol.22, Jan. 2004, pp.5-53. 